\title{
STABLE EQUIVALENCE OF DIFFERENTIABLE MANIFOLDS
}

\author{
BY BARRY MAZUR
}

Communicated by Edwin Moise, March 1, 1961

A natural question, of great generality, various special forms of which are often asked in differential topology, is the following:

Let $M_{1}, M_{2}$ be differentiable $n$-manifolds, $\phi: M_{1} \rightarrow M_{2}$ a continuous map which is a homotopy equivalence between $M_{1}$ and $M_{2}$. When is there a differentiable isomorphism

$$
\Phi: M_{1} \rightarrow M_{2}
$$

in the same homotopy class as $\phi$ ?

For example, there is the Poincare Conjecture which poses the question when $M_{1}$ is an $n$-sphere (see Smale [2], Stallings [3]).

I should like to suggest a certain simpleminded "stabilization" of the above question.

I shall say that $\Phi$ is a $k$-equivalence between $M_{1}$ and $M_{2}$, denoted:

$$
M_{1} \stackrel{\Phi}{\underset{\approx}{\rightleftarrows}} M_{2}
$$

for $k$ a non-negative integer, if $\Phi$ is a differentiable isomorphism between $M_{1} \times R^{k}$ and $M_{2} \times R^{k}$,

$$
\Phi: M_{1} \times R^{k} \underset{\approx}{\approx} M_{2} \times R^{k} .
$$

Now our original question may be reformulated as follows:

$\left(\mathrm{P}_{k}\right)$ If $\phi: M_{1} \rightarrow M_{2}$ is a homotopy equivalence, when is there a $k$-equivalence

$$
M_{1} \underset{\widetilde{(}}{\stackrel{\Phi}{\longrightarrow}} M_{2}
$$

$(\vec{k})$

in the same homotopy class as $\phi$ ? (I.e., such that

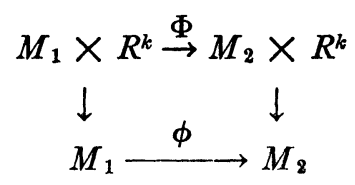

is homotopy commutative.) 
In the above terminology, a 0 -equivalence is simply a differentiable equivalence. It is easy to give examples of homotopically equivalent manifolds which are not 0 -equivalent, however are $k$-equivalent for some $k \geqq 0$. Thus, if $K^{3}$ is the complement, in $R^{3}$ of the Artin-Fox wild knot [1] the imbedding $\phi: K^{3} \rightarrow R^{3}$ provides a homotopy equivalence between the two manifolds. Nevertheless, they are not 0 -equivalent. After Stallings [3], $K^{3}$ and $R^{3}$ are 2-equivalent. In [8], a manifold $W^{4}$ is constructed which is a compact contractible 4-manifold whose boundary $\partial W^{4}$ is nonsimply connected, and such that int $W^{4}$ is not differentiable isomorphic to $R^{4}$. It is proved, however, that $W^{4} \times I \approx I^{5}$ and, in particular,

$$
\text { int } W^{4} \underset{(1)}{\approx} R^{4}
$$

More generally, it is a consequence of J. H. C. Whitehead's theory of Simple Homotopy Type $[6 ; 7]$ that if $A^{n}$ is a compact contractible differentiable $n$-manifold, there is a $k \geqq 0$ such that $A^{n} \times I^{k}$ is combinatorially isomorphic with $I^{n+k}$.

In the negative direction, Whitehead proves that for the lens spaces

$$
L_{i}=L(i, 7)=S^{3} /\left(Z_{7}\right)_{i}, \quad i=1,2
$$

even though $L_{1}$ is homotopically equivalent to $L_{2}, L_{1} \times \Delta^{k}$ is not combinatorially equivalent to $L_{2} \times \Delta^{k}$ for any $k \geqq 0$. This follows from his more general theorem:

Let $M_{1}, M_{2}$ be differentiable $n$-manifolds. They are of the same simple homotopy type if and only if $M_{1} \times \Delta^{k}$ is combinatorially equivalent to $M_{2} \times \Delta^{k}$.

Definition 1. A homotopy equivalence $\phi: M_{1}^{n} \rightarrow M_{2}^{n}$ between two differentiable $n$-manifolds will be called a $k$-differential homotopy equivalence if

$$
\phi^{*} T\left(M_{2}\right)+1_{k}=T\left(M_{1}\right)+1_{k}
$$

where $T(M)$ is the tangent bundle of the differentiable manifold $M$, $1_{k}$ is is the trivial $k$-plane bundle, $\oplus$ is the Whitney sum operation, and if $f: X \rightarrow Y$ is a continuous map between $X$ and $Y, E \rightarrow{ }^{\pi} Y$ a bundle over $Y, f^{*} E$ refers to the "pull back" bundle via the map $f$.

$M_{1}$ and $M_{2}$ will be called differentially homotopically equivalent if they are $k$-differentially homotopically equivalent for some $k \geqq 0$. Clearly a necessary condition for any affirmative solution of $\left(\mathrm{P}_{k}\right)$ is that the map $\phi: M_{1} \rightarrow M_{2}$ be a $k$-differential homotopy equivalence. 
This note is written as a partial statement of results to appear in a later paper. A sketch of the proof of one of the theorems is given. I am very thankful to John Milnor for discussions, for his sending me a copy of [5] which suggested the main theorem, and for his improvements of my presentation.

Theorem 1. Let $M_{1}, M_{2}$ be compact differentiable $n$-manifolds without boundary. Then $M_{1}$ and $M_{2}$ are differentially homotopically equivalent if and only if they are $k$-equivalent for $k \geqq n+2$.

Thus, to pass from questions involving 0 -equivalence to analogous questions involving $k$-equivalence for large $k$, is to pass from differential topology to homotopy theory. The problem, given two $(k+1)$ equivalent manifolds $M_{1}, M_{2}$, of determining whether or not they are $k$-equivalent seems to have vague formal similarities with the problem of descent of the groundfield in algebraic geometry and also with the Witt Group in the theory of quadratic forms. (Let $V_{1}, V_{2}$ be two algebraic varieties defined over a field $k$, which are birationally equivalent when considered over an extension field $K$. When are they birationally equivalent over $k$ ?)

There are also analogous notions of stable equivalence for other differentio-topological entities:

Definition 2. Two imbeddings $f, g: M \rightarrow W$ will be called $k$-isotopic if the imbeddings

$$
\begin{aligned}
& f_{k}: M \times R^{k} \rightarrow W \times R^{k}, \\
& g_{k}: M \times R^{k} \rightarrow W \times R^{k}
\end{aligned}
$$

defined by:

$$
\begin{aligned}
& f_{k}(m, r)=f(m), \\
& g_{k}(m, r)=g(m)
\end{aligned}
$$

are globally isotopic.

Definition 3. Let

$$
\begin{aligned}
& \alpha_{1}: G \rightarrow \operatorname{Aut}\left(M_{1}\right), \\
& \alpha_{2}: G \rightarrow \operatorname{Aut}\left(M_{2}\right)
\end{aligned}
$$

be differentiable actions of the group $G$ on the manifolds $M_{1}, M_{2}$. Then $\alpha_{1}$ and $\alpha_{2}$ are called $k$-equivalent if the "extended" actions $\alpha_{1}^{(k)}, \alpha_{2}^{(k)}$ of $G$ on $M_{1} \times R^{k}, M_{2} \times R^{k}$ obtained by letting $G$ act trivially on $R^{k}$ are differentially equivalent (in the sense that there is a differentiable isomorphism 
sending $\alpha_{1}^{(\boldsymbol{k})}$ to $\left.\alpha_{2}^{(\boldsymbol{k})}\right)$.

I expect that there are theorems analogous to Theorem 1, for these notions of $k$-equivalence, linking them to homotopy theoretic conditions also (for large enough $k$ ). There is a generalization of Theorem 1 to vector bundles:

Theorem 2. Let E, $F$ be differentiable k-plane bundles over compact $n$-manifolds without boundary, for $k \geqq n+2$.

Then $E$ is differentially homotopic to $F$ if and only if $E$ is isomorphic to $F$, as differentiable manifolds.

CoROLlaRY 1. Let $M_{1}^{n}, M_{2}^{n}$ be compact $n$-manifolds without boundary such that $\phi: M_{1}^{n} \rightarrow M_{2}^{n}$ is a homotopy equivalence. If $\eta_{1}^{\boldsymbol{k}}, \eta_{2}^{\mathbf{k}}$ are differentiable $k$-plane bundles over $M_{1}^{n}, M_{2}^{n}$ such that

$$
T\left(M_{1}\right)+\eta_{1}=\phi^{*} T\left(M_{2}\right)+\phi^{*} \eta_{2}
$$

and if $E_{i} \approx E\left(\eta_{i}\right)(i=1,2)$ are the total spaces of $\eta_{i}$, considered as differentiable manifolds, then $E_{1} \approx E_{2}$.

CoRollary 2. If $M_{1}^{n}, M_{2}^{n}$ are compact differentiable manifolds with boundary, and of the same homotopy type, if $U_{1}, U_{2}$ are open tubular neighborhoods of their "canonical" imbedding in $R^{n+k}(k \geqq n+2)$, then $U_{1} \approx U_{2}$.

Employing the ideas of Stallings for the proof of the Generalized Poincaré Conjecture, $n \geqq 5$, the following may be shown:

TheOREM 3. Let $W$ be a differentiable manifold without boundary, $\operatorname{dim} W \geqq 6$. Let $f: M \rightarrow W$ be an imbedding of $M$, a compact manifold without boundary, in $W$, which is a homotopy equivalence. Let $E=E(\xi)$, the total space of the differentiable vector bundle $\xi$, where $\xi=\nu(f) \oplus 1$, $\nu(f)$ being the normal bundle of the imbedding $f: M \rightarrow W$, and 1 is the trivial line bundle.

Then $E$ is combinatorially isomorphic with $W \times R$.

The conclusion of Theorem 2 concerns the differential structure of the unbounded total space of differentiable vector bundle.

For any $\xi$, a differentiable vector bundle over $M, E=E(\xi)$, the total space, let there be a Riemannian metric on $\xi$ in the sense of $[4$, p. 37], and call

$$
E(r)=\{x \in E \mid\|x\| \leqq r\}, \quad r>0 .
$$

Then $E(r)$ is a differentiable manifold with boundary $S(r)$ $=\{x \in E \mid\|x\|=r\}$. It is easily seen that int $E(r) \approx E$. It is a conse- 
quence of J. H. C. Whitehead's theory of Simple Homotopy Type that:

Theorem (Whitehead [7]). Let $E, F$ be k-plane bundles over compact differentiable n-manifolds without boundary, such that $k$ is sufficiently large $\left(k \geqq k\left(M_{1}, M_{2}\right)\right.$ where $k\left(M_{1}, M_{2}\right)$ is a constant depending upon $M_{1}$ and $M_{2}$ ) such that $E, F$ admit Riemannian metrics and $E$ is of the same differential homotopy type as $F$. Then $E(r)$ is combinatorially isomorphic with $F(r)$ if and only if $E(r)$ has the same simple homotopy type as $F(r)$.

The theorem of Whitehead stated above may be improved to fit this context as follows:

THEOREM 4. Under the situation of the previous theorem, one has: $E(r)$ is differentiably isomorphic with $F(r)$ if and only if $E(r)$ and $F(r)$ have the same simple homotopy type.

CoRollary. If $M_{1}^{n}, M_{2}^{n}$ are differentiable manifolds (compact, without boundary), of the same differential homotopy type, then $M_{1}^{n}$ is of the same simple homotopy type as $M_{2}^{n}$ if and only if

$$
M^{n} \times D^{k} \approx M_{2}^{n} \times D^{k}
$$

for large enough $k$.

The theorems stated above have generalizations to arbitrary manifolds, not necessarily compact without boundary; however the notion of differential homotopy type must be altered somewhat.

Sketch of the Proof of Theorem 2. Let $\mathfrak{M}$ denote the set of maps $f: M_{1} \rightarrow M_{2}$ satisfying these properties:

(1) $f: M_{1} \rightarrow M_{2}$ is an imbedding,

(2) $f$ (int $M_{1}$ ) is open in $M_{2}$,

(3) $f\left(M_{1}\right) \subseteq$ int $M_{2}$.

Such maps will be called open interior imbeddings.

INJECTIVE LIMITS. For any sequence of manifolds and maps,

$$
S: M_{1} \underset{f_{1}}{\rightarrow} M_{2} \underset{f_{2}}{\rightarrow} M_{3} \underset{f_{3}}{\rightarrow} \cdots, \quad f_{i} \in \mathfrak{M}
$$

a natural differential structure may be placed on the injective limit, Inj $\operatorname{Lim}(S)$, in an obvious manner.

If

$$
\begin{aligned}
& \phi: E \rightarrow F, \\
& \psi: F \rightarrow E
\end{aligned}
$$


are maps, $\phi, \psi \in \Re$, consider the sequence

$$
S(\phi, \psi): E \underset{\phi}{\rightarrow} F \underset{\psi}{\rightarrow} E \underset{\phi}{\rightarrow} \cdots
$$

obtained by iteration. Define

$$
X(\phi, \psi)=\operatorname{Inj} \operatorname{Lim} S(\phi, \psi)
$$

considered as a differentiable manifold.

If $f: E \rightarrow E$ is a map, $f \in \mathfrak{M}$, consider the iterated sequence

$$
S(f): E \underset{f}{\operatorname{fa}} \underset{f}{\rightarrow} \underset{f}{\rightarrow} \cdots
$$

Define $X(f)=\operatorname{Inj} \operatorname{Lim} S(f)$.

It is tautological that

$$
X(\phi \circ \psi) \approx X(\phi, \psi) \approx X(\psi \circ \phi) .
$$

Definition 3. Let $\nu: E \rightarrow E, \nu \in \Re$.

Then $E$ will be called $\nu$-movable if for all $\rho: E \rightarrow E, \rho \in M$, which are homotopic to $\nu$, and $\alpha: E \rightarrow E$ an automorphism of $E$ homotopic to the identity automorphism, there exists an automorphism $\beta: E \rightarrow E$ homotopic to the identity, such that

$$
\begin{array}{r}
E \stackrel{\nu}{\rightarrow} E \\
\alpha \downarrow \quad \beta \downarrow \\
E \stackrel{\rho}{\rightarrow} E
\end{array}
$$

is commutative.

$E$ is called movable if it is $\nu$-movable for some $\nu$ homotopic to the identity.

Proposition 1. Let $E$ be movable and let $f: E \rightarrow E, f \in M$, be homotopic to the identity; then:

$$
X(f) \approx \operatorname{int} E .
$$

Proposition 2. Let $E$ be a differentiable k-plane bundle over a compact n-manifold without boundary, such that $k \geqq n+2$. Let $r>0$. Then $E(r)$ is movable.

Proposition 2 comes essentially from the following technical lemma:

Lemma. Let $E$ be a differential vector bundle over a compact manifold $M$. Let $i: M \rightarrow E$ denote the zero cross-section, and $W$ a manifold. Let

$$
f, g: E \rightarrow W,
$$

$f, g \in \mathbb{N}$ 
such that

$$
f \circ i=g \circ i \text {. }
$$

Then there is an automorphism

and

$$
\begin{aligned}
& \alpha: W \rightarrow W, \\
& \text { a real number } r>0,
\end{aligned}
$$

a linear bundle automorphism

$$
\begin{gathered}
\lambda: E \rightarrow E \\
\pi \searrow{ }_{M}{ }^{\swarrow \pi}
\end{gathered}
$$

such that $\lambda: E(r) \approx E(r)$ for which

$$
\begin{array}{ll}
E(r) \underset{f}{\rightarrow} W & \\
\downarrow \lambda & \downarrow \alpha \\
E(r) \underset{g}{\rightarrow} & W
\end{array}
$$

is commutative.

Proposition 3. Let $E, F$ be differentiable $k$-plane bundles over compact $n$-manifolds without boundary, $k \geqq n+2$.

If $E$ and $F$ are differentiably homotopic, there exist maps

$$
\phi: E(r) \rightarrow F(r) \quad \psi: F(r) \rightarrow E(r), \quad \phi, \psi \in \mathfrak{N}, r>0
$$

such that $\phi$ and $\psi$ are two-sided homotopy inverses.

The proof of Theorem 2 follows from these three propositions. For the hypotheses of Theorem 2 are the hypotheses of Proposition 3. Therefore, we are guaranteed a $\phi, \psi$ as in Proposition 3, and

$$
\phi \circ \psi \sim 1_{F(r)}, \quad \psi \circ \phi \sim 1_{E(r)} .
$$

By Propositions 1 and 2,

$$
\begin{aligned}
& X(\phi \circ \psi) \approx \text { int } F(r) \approx F, \\
& X(\psi \circ \phi) \approx \text { int } E(r) \approx E .
\end{aligned}
$$

By (III)

$$
F \approx X(\phi \circ \psi) \approx X(\psi \circ \phi) \approx E
$$

proving the theorem.

The Hauptvermutung. It is a result of Whitehead that the lens 
spaces $L_{1}, L_{2}$ are not of the same simple homotopy type. Thus $L_{1} \times \Delta^{k}$ is combinatorially inequivalent to $L_{2} \times \Delta^{k}$ for any $k \geqq 0$.

However, $L_{1}$ and $L_{2}$ are of the same differential homotopy type. (They are of the same homotopy type [7], and all orientable 3manifolds are parallelizable, so they have the same differential homotopy type.)

It is a consequence of Theorem 1 that $L_{1} \times R^{2}$ is differentiably isomorphic with $L_{2} \times R^{5}$.

Using these results, Milnor has constructed finite complexes $K_{1}^{8}$, $K_{2}^{8}$ which are topologically isomorphic yet are combinatorially inequivalent, thus contradicting the Hauptvermutung.

$$
K_{i}^{8}=\left(L_{i} \times \Delta^{5}\right) \cup C\left\{\partial\left(L_{i} \times \Delta^{5}\right)\right\}, \quad i=1,2
$$

where $C\{X\}$ denotes the cone over $X$. That $K_{1}^{8}$ is topologically isomorphic to $K_{2}^{8}$ can be seen since $K_{i}^{8}$ is (topologically) the one-point compactification of $L_{i} \times R^{5}(i=1,2)$.

\section{REFERENCES}

1. E. Artin and R. Fox, Some wild cells and spheres in three-dimensional space, Ann. of Math. vol. 49 (1948) pp. 979-990.

2. S. Smale, The generalized Poincaré conjecture in dimensions greater than four, Ann. of Math., to appear.

3a. J. Stallings, A characterization of Euclidean space of dimension greater than four, Lecture at the Harvard-M.I.T.-Brandeis Colloquium, to be published.

3b. —, Polyhedral homotopy-spheres, Bull. Amer. Math. Soc. vol. 66 (1960) pp. $485-488$.

4. J. Milnor, Notes on differential topology, Princeton University, Fall term, 1958.

5. - Differential structures on $S^{n} \times R^{k}$, unpublished.

6. J. H. C. Whitehead, Simplicial spaces, nuclei and m-groups, Proc. London Math. Soc. vol. 45 (1939) pp. 243-327.

7. - Simple homotopy types, Amer. J. Math. vol. 72 (1950) pp. 1-57.

8. B. Mazur, A note on some contractible 4-manifolds, Ann. of Math. vol. 73 (1961) pp. 221-228.

HARVARD UNIVERSITY 